\title{
Frédéric Le Play y su círculo de reforma social
}

\author{
José Ignacio Garrigós Monerris
}

Universidad de Alicante. Departamento de Sociología

\section{Resumen}

Frédéric Le Play fue uno de los pioneros de la sociología y un impulsor decisivo del método científico en las ciencias sociales en el siglo XIX francés, marcado por la inestabilidad social que las revoluciones políticas y el nuevo orden social, traído por la revolución industrial, caracterizó. La preocupación por la paz, la estabilidad y el progreso social era compartida por personalidades de diferentes tendencias políticas que se unieron en torno a la idea de la necesidad de realizar reformas sociales para alcanzar una sociedad estable. En los años treinta y cuarenta, Le Play frecuentaba ciertos salones en los que se discutía acerca de la reforma social, es decir, de los cambios que debían realizarse en la sociedad para evitar los desórdenes y las injusticias, lo que le da la oportunidad de compartir sus desvelos por la paz social, nacidos ante la visión de los hechos sangrientos que produjo la revolución de 1830. En estos salones, Le Play estableció lo que podríamos llamar «su círculo intelectual». Este círculo intelectual, estos compañeros de inquietudes sociales, ven en Le Play y en sus observaciones una voz apta que les guíe en una política de reformas sociales basada en principios científicos. En este artículo se pretende describir este círculo intelectual y mostrar la influencia que tuvo para que Le Play desarrollara un método científico para la sociedad. La reflexión sobre la cuestión social afectó, sin duda, a la política social y, por ende, al desarrollo de la sociología.

Palabras clave: Frédéric Le Play, historia de la sociología, círculo intelectual, reforma social.

\section{Abstract. Frédéric Le Play and his circle of social reform}

Frédéric Le Play broke new ground in sociology and was a driving force behind the use of scientific method in the Social Sciences. He lived in the France of the $19^{\text {th }}$ century, which was marked by social unrest, a result of the political revolutions and the new social order brought about by the industrial revolution. His concern for peace, stability and social progress was shared by eminent people of his day, of varying political persuasions, who were brought together by the belief that social reforms were necessary in order to create a stable society. Throughout the thirties and forties, Le Play was a frequent visitor to various salons where social reform was the issue of the day, that is to say, how society should be changed in order to prevent unrest and injustice, and this enabled him to share his struggles to achieve social peace, which first began after witnessing the bloody events of the 1830 revolution. It was in these salons, that Le Play established what we might call his intellectual circle. This intellectual circle, composed of like-minded people concerned about society, see in Le Play and his observations a fitting voice to guide them in a policy of social reform based on scientific principles. The aim of this article is to describe this intellectual circle and show how its influence 
helped Le Play develop a scientific method for society. It cannot be doubted that discussion of the social question affected social policies and, thus, the development of sociology.

Key words: Frédéric Le Play, history of sociology, intellectual circle, social reform.

\section{Sumario}

\section{Introducción 4. Los últimos años}

\section{Los primeros salones. Le Play se presenta Bibliografía}

en sociedad

3. Le Play es requerido como experto en

cuestiones sociales

\section{Introducción}

Pierre-Guillaume-Frédéric Le Play (La Rivière-Saint-Saveur, Calvados, 1806París, 1882) es una figura clave en el proceso de formación de las ciencias sociales. Aunque ingeniero de minas de formación, Le Play intentó trasladar el método metalúrgico a las ciencias sociales para construir un método científico de la sociedad. De este modo, creó un método de investigación con vocación científica, primando la observación sobre las ideas preconcebidas. Propuso un nuevo paradigma de investigación en el cual el propio investigador definía los principios que iba a estudiar y recogía sus propios datos. Eligió como objeto de su estudio a familias tipo de cada sociedad, confeccionó una guía práctica para realizar estudios sociales, combinó indicadores cuantitativos y cualitativos y elaboró teorías sociológicas conectando conceptos como la movilidad social y la estructura familiar, la moralidad y el éxito económico o la estructura familiar y el tipo de trabajo.

Por otro lado, Le Play vivió muy de cerca las transformaciones sociales y los acontecimientos históricos de su país y de su tiempo. No tardó en conocer el éxito y en obtener el reconocimiento social a su labor tanto como ingeniero de minas, primero, como científico social, después. Fue nombrado catedrático de la Escuela de Minas, inspector general de Minas de Francia, comisario general de las Exposiciones Universales parisinas de 1855 y 1867 y consejero de estado y senador imperial bajo Napoleón III. Fundó la Société d'Économie Sociale, el primer centro de investigación social privado de Francia, y fue distinguido como gran oficial de la Legión de Honor. Entre sus amigos se encontraba la elite política e intelectual francesa. Su preocupación por la paz social en un siglo repleto de revueltas y caracterizado por la llegada de la sociedad industrial, le llevó a estudiar las distintas sociedades europeas y a interesarse por las condiciones de la estabilidad social. 
En este artículo no se quiere abordar el pensamiento sociológico de Le Play ni sus aportaciones metodológicas a las ciencias sociales. A lo largo de estas líneas se pretende reflejar la importancia que Le Play tuvo como experto en cuestiones sociales en Francia, principalmente en los últimos años de la Monarquía de Julio y en la Segunda República, entre un heterogéneo grupo de intelectuales y personalidades preocupadas por la paz social. En torno a Le Play se formó un "círculo reformador" compuesto por personas de diferentes tendencias políticas unidas por la idea de la necesidad de realizar reformas sociales para alcanzar la estabilidad de la sociedad. De este modo, se persigue poner de manifiesto, a través de las personalidades más destacables, el relieve social que Le Play alcanzó en la sociedad de su tiempo, pues es algo que afectó, sin duda, a los orígenes de la sociología como ciencia.

Es evidente que un determinado contexto histórico y social produce unas determinadas biografías, unos autores peculiares que elaboran una obra, la cual, a su vez, influye en la propia vida del autor y en su contexto social e intelectual. Para poder interpretar y valorar adecuadamente el trabajo de cualquier teórico o investigador, es imprescindible situar sus ideas, sus aportaciones metodológicas y sus propias vidas en su contexto histórico y social, y debemos, por tanto, acercarnos todo lo posible a los problemas de su tiempo y a sus experiencias vitales, que son las que conforman su manera de ver el mundo.

Por todo ello y partiendo de estos presupuestos, en este artículo se intenta hacer una sociología narrativa, que cuente un período de la vida del ingeniero de minas Frédéric Le Play determinante para su posterior total dedicación a la ciencia social. Al mismo tiempo, se hace un recuento de aquellas personas que acompañaron a Le Play en su camino hacia la sociología y se intenta, así, transmitir el clima de preocupación por lo social que caracterizó a ciertas elites. Tal preocupación se debía al nuevo orden social, resultado de la reciente sociedad industrial que produce tipos inéditos de relaciones muy complejas que ya no entran en el molde simplista de la sociedad anterior. En los años treinta y cuarenta del siglo XIX, Le Play forma parte de un grupo de personas destacadas de la sociedad francesa preocupadas por la paz social, que desean trabajar en pos de una sociedad estable, alejada de las convulsiones sociales que ocasionaron las revoluciones de 1830 y 1848 . Es éste el momento en el que vamos a detenernos. A partir de 1856 y sobre todo de 1870, Le Play fundará su propia escuela, su propio círculo y tendrá sus discípulos, sin embargo este artículo se va a centrar en los años de formación, de inquietud por la cuestión social e intentará profundizar en los círculos, un tanto informales, a los que perteneció Le Play, compuestos de personalidades que reflexionan acerca de la cuestión social. Se trata de círculos no institucionalizados ni formalizados pero que, sin embargo, existieron y tuvieron su importancia en lo que será el diseño de una sociedad estable, además de marcar el camino de Le Play hacia la ciencia social.

La participación de Le Play en estos círculos puede diferenciarse en dos etapas cuya frontera estaría en el mes de febrero de 1848. Durante los años de la Monarquía de Julio, Le Play se da a conocer como un destacado ingeniero en los círculos sociales de París, lo que le da la oportunidad de compartir sus 
desvelos por la paz social, nacidos ante la visión de los hechos sangrientos que produjo la revolución de 1830 , con un grupo de personalidades muy destacadas. De hecho, en los últimos años de la Monarquía de Julio, Le Play se integra en el proyecto de reforma social de Tocqueville y Lanjuinais. A partir de febrero de 1848, Le Play colabora con la Segunda República con la intención de demostrar que su método monográfico podría resolver los problemas sociales. En estos años, Le Play es una figura conocida de la sociedad francesa y sus amigos, su círculo, le animan para que se dedique por entero a la cuestión social.

\section{Los primeros salones. Le Play se presenta en sociedad}

El mes de julio de 1830 se caracterizó en Francia por la revolución que destituyó la monarquía autoritaria de Carlos X e implantó la monarquía burguesa y liberal de Luis Felipe I. Frédéric Le Play se encontraba, en estos meses, convaleciente de un accidente de laboratorio, pero se sintió muy consternado por las escenas de sangre, odio y dolor que le relataban las personas que le visitaban y no podía dejar de recordar las impresiones de paz y armonía social que tenía frescas del viaje a Alemania que realizó como estudiante de ingeniería junto a su compañero y amigo Jean Reynaud. Fue en ese momento, aún enfermo, cuando decidió dedicar todos sus esfuerzos a remediar, en la medida de lo posible, los males sociales que azotaban Francia (Le Play, 1877-79, I, p. 41):

Fue entonces, en efecto, que tomé la resolución de remediar, tanto como me fuera posible, las llagas abiertas de mi país. Formé el propósito de dedicar cada año, seis meses de viaje a mis estudios metalúrgicos, mezclándolos con los de las familias y las sociedades. Cumplí fielmente mi propósito.

Efectivamente, como el mismo Le Play señala, no faltó a su propósito. El invierno lo pasaba en Francia trabajando como secretario en la publicación de los Annales des Mines y la Statistique de l'Industrie minérale, daba en la Escuela de Minas, desde 1840, la clase de metalurgia y redactaba las memorias de sus misiones. Durante los seis meses de mejor tiempo se dedicaba a viajar. Pero sus viajes tenían una finalidad bien precisa: la mayoría de las veces, como ingeniero, estaba encargado de misiones oficiales y, allí donde iba, entraba en contacto con los patronos, los obreros y las personalidades públicas. También tomaba buena nota de los procedimientos técnicos que hacían prosperar a las empresas, al tiempo que buscaba afanosamente las instituciones que se encargaban de asegurar a las familias el bienestar y la satisfacción moral, la unión y la concordia de las distintas clases sociales y la confianza en el porvenir y la estabilidad de los pueblos. Además, Le Play quiso aplicar el mismo método al examinar cuestiones metalúrgicas y cuestiones sociales.

A lo largo del reinado de Luis Felipe I duque de Orleans, conocido como la Monarquía de Julio (1830 -1848), Frédéric Le Play va adquiriendo una cierta notoriedad en los círculos que debaten la política industrial y social de su 
tiempo. Su trabajo en la Comisión Permanente de Estadística de la Industria Minera, así como en los Annales des Mines, le evidencia como un ingeniero de minas de talento. El conde Adrien de Gasparin (1783-1862), agrónomo y miembro de la Academia de Ciencias, destaca el informe anual de la Comisión de Estadística que Le Play realizó en el año 1835 y, por otro lado, el ministro de Comercio, Hyppolite Passy (1793-1880), le envía a Inglaterra, como ingeniero de minas, en el año 1836, para que estudie la industria de la hulla y del hierro. El mismo afán de desarrollar una política positiva, basada en el conocimiento objetivo de los hechos y de las opiniones para orientar adecuadamente el sentido de los asuntos públicos, fue lo que movió al ministro de Interior Montalivet (1801-1880) a permitir, en el año 1831, que Tocqueville partiera a los Estados Unidos de América junto a su colega y amigo Gustave Beaumont (1802-1866), con el fin de estudiar el sistema penitenciario norteamericano. Fruto de este viaje fue la publicación, en 1833, de la obra Du système pénitentiaire aux États-Unis et de son application en France, además de la conocida De la démocratie en Amérique (1835-1840).

Las capacidades profesionales de Le Play no pasan, por tanto, desapercibidas. Adolphe Thiers (1797-1877), por entonces uno de los jefes de la oposición, le propone ser candidato en las elecciones legislativas de 1838, pero Le Play rechaza tal ofrecimiento.

Frédéric Le Play frecuentaba algunos de los salones parisinos que en esta época servían como lugares de encuentro donde, entre otras cosas, se reflexionaba sobre cuestiones culturales y sociales. De este modo, sus investigaciones sociológicas fueron conocidas en los ambientes políticos e intelectuales de los últimos años de la Monarquía de Julio. Dos eran los salones que Le Play visitaba con más asiduidad ${ }^{1}$ : el de Mathilde Bonaparte y el de su amigo Jean Reynaud.

La princesa Mathilde (1820-1904), hija de Jerôme Bonaparte (1784-1860), conoció a Le Play cuando éste trabajó para su marido, el príncipe ruso Anatol Demidoff (1813-1870), importante terrateniente y destacada personalidad de la aristocracia europea, reorganizando sus minas de los Urales en el año 1844. En 1846, el matrimonio entre la francesa y el ruso se había roto y ella volvió a París. En este salón, Le Play se relaciona con los círculos bonapartistas.

Por otro lado, en el salón de su antiguo amigo y compañero de estudios Jean Reynaud (1806-1863), Le Play se encuentra con personalidades como François Arago (1786-1853), profesor de geometría descriptiva en la Escuela Politécnica entre los años 1809 y 1830, director del Observatorio de París, donde enseña astronomía, de 1813 a 1846 y secretario perpetuo de la Academia de las Ciencias desde el año 1830; como Jean-Baptiste Dumas (18001884), destacado químico, miembro de la Academia de Ciencias desde 1832 y secretario perpetuo desde 1868 que formará parte de la Asamblea Legislativa en 1849 y será ministro de Agricultura y Comercio entre los años 1850 y 1851; como el conde Charles Forbes de Montalembert (1810-1870), que será

1. Véase, Antoine SAVOYE, 1989, p. 23. 
jefe de los católicos liberales hasta 1857; como Augustin Cochin (1823-1872), estudioso de cuestiones relativas a la economía social y política, sobre todo del problema del pauperismo, que publicará varios escritos sobre la obra de Le Play, que estuvo afiliado al partido católico liberal convirtiéndose en teniente de alcalde del distrito X de París en 1853 y que entró, en el año 1864, en la Academia de Ciencias Morales y Políticas y, en fin, como los parlamentarios Alexis de Tocqueville (1805-1859) y el vizconde Victor Ambroise de Lanjuinais (1802-1869). Con todos ellos Le Play proyecta reformas sociales. Tocqueville fue diputado y magistrado de 1839 a 1848 situándose en una posición centrista y mostrándose partidario de reformas democráticas. En el año 1849 fue ministro de Asuntos Exteriores y, tras el golpe de Estado de Napoleón III, firmó una demanda contra éste, por lo que fue encarcelado. Su celebérrima obra De la démocratie en Amérique le valió el ingreso en la Academia de Ciencias Morales y Políticas el año 1836 y en la Academia Francesa en 1841. Por su parte, el vizconde de Lanjuinais, amigo de Tocqueville, fue elegido diputado por el Loira Interior en 1838, 1839, 1842 y 1846 . Formó parte del centro izquierda, aunque, posteriormente, se inclinó hacia los conservadores. Será nombrado ministro de Agricultura y Comercio en el gabinete presidido por Odilon Barrat (2 de junio de 1842). Lanjuinais se opuso vivamente al golpe de Estado de Napoleón III, por lo que fue arrestado, aunque pronto recobró la libertad.

Tocqueville y Lanjuinais vinculan a Le Play a su proyecto de formar «una asociación de algunos hombres de talento y corazón, que se mantengan apartados de las intrigas [...] trabajando los asuntos por ellos mismos». Tocqueville opina, en las postrimerías de la Monarquía de Julio, año $1846^{2}$, que «existe una posición a tomar, grande y patriótica, fuera de la política diaria con respecto al pueblo propiamente dicho y es de este lado donde se encuentra el futuro más grande». Por ello, está seguro de que "un partido que se diera por misión principal trabajar política y activamente por el bienestar moral y material de las clases inferiores, sin alimentar sus prejuicios ni inflamar sus pasiones, asumiría un papel a la vez lleno de novedad y de grandeza». Entre las fuerzas políticas francesas, Tocqueville encuentra un espacio por conquistar donde quiere instalar la «joven izquierda» integrada por un pequeño grupo de parlamentarios que, frente «al desprecio egoísta e ignorante de la mayoría conservadora» y «a los sueños y a las pasiones de los utópicos», luchará contra la atonía política e iniciará reformas constructivas. Le Play es el experto en materia social de este incipiente grupo, pues son conocidas las observaciones sociales que, con intención científica, estaba realizando a lo largo de sus viajes por Europa.

De hecho, de 1844 a 1848, se reunían todas las semanas, unas veces en casa de Le Play y otras en la de Reynaud o en la de Lanjuinais, para trabajar juntos en la elaboración de la reforma social ${ }^{3}$. Aunque provenían de distintas

2. Carta de Tocqueville a Jules-Armand Dufaure de 1846, citada por A. JARDIN, Alexis de Tocqueville, París: Hachette, 1984, p. 380 y recogida por A. SAVOYE, 1989, p. 23.

3. Le Play, 1877-79, I, p. 422. 
tendencias políticas, todos intentaron influir en los representantes de los diversos partidos del Parlamento para acordar los principios fundamentales de la reforma social. De este modo, Montalembert se ocupó de los pares de Francia y Victor Lanjuinais y Alexis de Tocqueville lo hicieron de los diputados. Treinta años después, Le Play recuerda melancólicamente aquellos esfuerzos de buena voluntad tan desinteresados (Le Play, 1877-79, I, p. 423):

Casi todos aquellos hombres han muerto. Los supervivientes tienen, pues, el deber de recordar esta época a los contemporáneos que conservan el espíritu de paz y de unión y no desesperan de la salvación de la patria. Mi viejo amigo Albert de Saint-Léger y yo recordamos a menudo que, en enero de 1848, en una de las agradables veladas de Jean Reynaud en que se agitaba la cuestión de la reforma, decía el señor Louis Blanc: «Evitemos las revoluciones: la experiencia nos enseña que el pueblo no gana nada con ellas; antes al contrario, le han hecho siempre menos feliz y menos libre».

Por medio de la reforma social se pretendía llevar a cabo las transformaciones necesarias capaces de evitar las revoluciones y las injusticias sociales. En realidad, en estos años, las ideas de estos círculos reformistas no llegan a materializarse en instituciones concretas, pero sí que nos muestran el interés que tenían algunos miembros destacados de la sociedad francesa por asegurar la paz y la estabilidad social. La corrupción del poder y el sufrimiento de la población debían eliminarse reformando la sociedad, pero esta reforma, ante todo, debía ser pacífica. Le Play (1877-79, I, p. 423), años después, lo apunta claramente:

Estábamos todos de acuerdo en un punto esencial: la ejecución de la reforma social era, por excelencia, la obra de la paz; y ella excluía formalmente todo recurso a la violencia.

\section{Le Play es requerido como experto en cuestiones sociales}

En el mes de febrero de 1848 estalla la revolución, Luis Felipe I es destronado y se instaura la Segunda República. La preocupación que Le Play había experimentado por los aspectos sociales en la revolución de julio de 1830, y que le había movido a estudiar las instituciones sociales y la organización de la sociedad de todos los pueblos que visitó, se acrecentó con esta revolución del año 1848. Le Play no se había comprometido con el poder orleanista y, por otro lado, ya era reconocido por sus estudios acerca de las cuestiones obreras y por sus ideas reformistas.

Lazare Hippolyte Carnot (1801-1888), parlamentario de la izquierda, que conocía el trabajo de Le Play y que fue ministro de Instrucción Pública y Cultos del gobierno provisional de 1848, nombró a Jean Reynaud subsecretario de Estado de ese ministerio. Reynaud, a su vez, nombró a Le Play miembro de la Comisión para los Trabajadores —o Comisión de Luxemburgo, tal como fue conocida - a título de experto. Le Play participó en el gobierno provisio- 
nal de 1848 para poder demostrar su convencimiento de que sus observaciones sociales y el método monográfico de las familias obreras que elaboró, podrían resolver los problemas sociales y restaurar la paz y la prosperidad. Estaba totalmente seguro de que las diferencias políticas podían resolverse estudiando la evidencia recogida en su método. Así, escribe (Le Play, La réforme sociale en France, edición de 1878, I, p. V):

Ha llegado el momento, para Francia, de sustituir las luchas estériles, suscitadas por el vicio del antiguo régimen y por el error de las revoluciones, por un entendimiento fecundo, fundado sobre la observación metódica de los hechos sociales.

Esta comisión del gobierno tenía carácter consultivo. Su objetivo consistía en el estudio de las condiciones de los trabajadores y estuvo presidida por el socialista Louis Blanc (1811-1882), que era ministro de Trabajo del gobierno republicano provisional. La Comisión estaba integrada por trabajadores, empleadores y expertos, y, en ella, Le Play expuso la situación de los mineros del Hartz que había observado en su primer viaje a Alemania, como ejemplo de estabilidad social. Se discutieron varias de las propuestas de Le Play para reformar las condiciones laborales de los trabajadores y, finalmente, la Comisión recomendó una reducción del número de horas de trabajo semanales y el establecimiento de comités — conseils de prud'hommes - para resolver las disputas entre trabajadores y empleadores.

Le Play coincidía con los socialistas Louis-Auguste Blanqui (1805-1881) y Étienne Cabet (1788-1856) en la necesidad de controlar los mecanismos del mercado y crear cooperativas de trabajo y asociaciones voluntarias, incluyendo sindicatos. Valoraba estas instituciones porque, a sus ojos, facilitarían el acuerdo entre trabajadores y empleadores y, por tanto, fomentarían la estabilidad social.

El gobierno conservador salido de las primeras elecciones generales para una Asamblea Nacional Constituyente celebradas el 23 de abril de 1848, clausuró, el 25 de junio de ese año, los Talleres Nacionales -Ateliers Nationaux-, creados por Louis Blanc durante el gobierno provisional para mitigar las cifras de desempleados, lo que provocó la insurrección de los trabajadores de París. El ministro de la Guerra, Eugène Cavaignac (1802-1857), reprimió duramente esta insurrección causando más de diez mil muertos y forzando la huida de Louis Blanc a Inglaterra.

Estos acontecimientos evidenciaron la inestabilidad de la sociedad y reabrieron la preocupación por la situación social. François Arago, que se había encargado de los ministerios de Marina y de Guerra en el gobierno provisional y que pasará a la historia por abolir la esclavitud en las colonias francesas, estaba consternado por el rumbo que tomaba la reciente Segunda República, que él había dirigido desde sus comienzos. Por ello, a través de Reynaud, solicitará a Le Play, su antiguo alumno de la Escuela Politécnica, que aporte argumentos para fundar una política social reformadora capaz de alcanzar y mantener la paz social. 
Los republicanos Reynaud y Arago no son los únicos que, a consecuencia de los sucesos de junio de 1848, reflexionan sobre la condición de los obreros y de las reformas a realizar en la sociedad francesa. Personalidades de todos los medios políticos están preocupadas por estos mismos aspectos y ven, en Le Play y en sus observaciones, una voz apta que les guíe en una política de reformas sociales basada en principios científicos. Adolphe Thiers, por entonces jefe del partido del Orden que dirigió la represión de junio, y Alphonse de Lamartine (1790-1869), que fue ministro de Asuntos Exteriores en el gobierno provisional, además de los ya citados Tocqueville, Lanjuinais, Dumas, Montalembert y Cochin, también animaron a Le Play en su tarea de observación social y destacaron su trabajo como «la obra de salvación pública» (Le Play, 1877-79, I, p. 426). El círculo de Le Play se fue ampliando con notables provenientes de otros campos, como Jacob Rothschild (1792-1868), el menor de los cinco hijos del fundador de la familia alemana de banqueros, Mayer Amschel (1744-1812), que inauguró la casa de París en 1812 y que también fue cónsul de Austria en 1822, o como el abate Félix Dupanloup (1802-1878), que será obispo de Orléans desde 1849 y jefe de los católicos liberales bajo el reinado de Napoleón III. Asimismo, pertenecen a este círculo los ya mencionados Carnot y el conde de Gasparin, par de Francia y ministro del Interior en la Monarquía de Julio. También se encontraban el economista, matemático y político Charles Dupin (1784-1873) y el escritor y crítico literario CharlesAugustin Sainte-Beuve (1804-1869). Dupin fue ministro de Marina en 1833 y, en 1848, formó parte de la Asamblea Constituyente y, luego, de la legislativa. Es uno de los creadores de los servicios estadísticos franceses y fue el autor del informe por el cual la Academia de Ciencias otorgó, en enero de 1856, el premio Montyon de estadística a la obra de Le Play, Les ouvriers européens. Sainte-Beuve era un habitual en el salón de la princesa Mathilde, donde conoció a Le Play. Después de la publicación de su única novela, Volupté (1834), se alejó del romanticismo y sufrió una crisis ideológica, consecuencia, en parte, de la decepción que le produjeron el saint-simonismo y el catolicismo liberal de Lamennais (1782-1854), hacia los que se había sentido atraído. En el año 1844 ingresó en la Academia Francesa adoptando una postura anticlerical y combatiendo ardientemente al abate Dupanloup. Fue uno de los más destacados intelectuales que se adhirieron al Segundo Imperio, siendo nombrado senador el año 1865. Escribió muy entusiasta y favorablemente sobre Le Play y su obra.

En un período en el cual los historiadores, los filósofos y hasta los literatos como Balzac, Stendhal, Sainte-Beuve, Tocqueville o Hyppolite Taine, pretendían expresar en sus libros únicamente la realidad observada a través de procedimientos objetivos que emularan a las ciencias naturales, Le Play era muy tenido en cuenta, precisamente, por su procedimiento científico, por su tendencia a aplicar a todo un mismo método de investigación.

François Arago insistió más que nadie para que Le Play revisara sus observaciones sociales y las preparara para una gran publicación. Sin embargo, cuando Le Play las publicó, el año 1855, Arago ya había fallecido. Como el propio Le Play señala (1877-79, I, p. 42), Arago reconocía que la solución a los 
problemas sociales no se hallaría ni en el comunismo organizado por el Estado, ni en el laissez-faire que predicaban los economistas. Le Play, que tenía en mente realizar una gran obra sobre la metalurgia que titularía L'Art métallique au XIX siècle y que tenía gran apego a sus estudios metalúrgicos, después de algunas vacilaciones, acabó abandonando su cátedra de la Escuela de Minas ${ }^{4}$ y entregándose a perfeccionar sus observaciones y conclusiones sobre la organización de la sociedad para alcanzar la paz social. También Adolphe Thiers insistió mucho en que abandonara sus estudios de metalurgia diciéndole que, «en circunstancias tan difíciles, el primer deber de un francés era "fortalecer las convicciones debilitadas" " (Le Play, 1877-79, I, p. 426). De este modo, dice Le Play (1877-79, I, p. 426 y 433), comenzó la tercera etapa de su vida, dedicada por entero al estudio de la reforma social.

La vuelta al orden que se produce, sobre todo, a partir del golpe de Estado del 2 de diciembre de 1851 de Luis Napoleón Bonaparte (1808-1873), dispersa el círculo reformador que se había constituido alrededor de Le Play. A pesar de sentirse solo, no por ello abandona la elaboración de su trabajo de observación social.

Aunque el círculo que se formó alrededor de él se disolvió, Le Play extrae la reconfortante conclusión de que personas con diferentes ideologías son capaces de unirse para realizar, o al menos para proponer, reformas sociales. Es evidente que la idea de la reforma social, es decir, la búsqueda de la paz, de la estabilidad y de la prosperidad de la sociedad marcó y dirigió, de alguna manera, el desarrollo de la política social y de la sociología.

Luis Napoleón ya conocía, por los medios bonapartistas, la brillante labor científica, administrativa y organizadora que Le Play había desempeñado en las minas del príncipe Demidoff, quien, por otro lado, fue testigo en el matrimonio de Le Play y Augustine Fouache el año 1840. Esto, junto con el renombre que Le Play había alcanzado en la sociedad francesa, fue decisivo para que el emperador le nombrara comisario general de la Exposición Universal de París de 1855. El éxito fue enorme y Le Play fue designado, en 1856, consejero de Estado. El Consejo de Estado era un órgano consultivo, no legislativo, y su papel era preparar leyes que serían votadas en la Cámara de diputados.

Además, Le Play publica, el año 1855, sus observaciones sociales, realizadas según un método científico comprensible, bajo el título de Les ouvriers européens 5 , en las prensas imperiales, con cargo a los fondos oficiales. Le Play pasa, de este modo, a convertirse en asesor privado de Napoleón III. El mes de abril de 1856, coincidiendo con su cincuenta cumpleaños, inaugura la Société d'Économie Sociale ${ }^{6}$ y, a través de ella, se esfuerza por precisar las conclusiones prácticas de sus

4. De todos modos, no abandonará definitivamente su cátedra hasta 1856 , año en que es nombrado consejero de Estado.

5. El título completo es: Les ouvriers européens. Études sur les travaux, la vie domestique et la condition morale des populations ouvrières de l'Europe, et leur relations avec les autres classes, precedées d'un exposé de la méthode d'observation.

6. Nombre por el que se conoce a la Société International d'Études Pratiques d'Économie Sociale. 
investigaciones sociológicas e, igualmente, procura entrar en contacto con personalidades de todas las tendencias del mundo cultural, de los medios políticos y de los económicos para transmitirles sus ideas. De este modo, vemos a Le Play al lado del príncipe Jerôme Bonaparte, primo hermano de Napoleón III, que solicita su adhesión a la Société d'Économie Sociale el 12 de noviembre de $1856^{7}$ o, aconsejando, desde el año 1858, al liberal Émile Ollivier (1825-1913), quien se convertirá en ministro de Justicia y Cultos en $1870^{8}$. El primer presidente de la Société será el doctor y reputado investigador social Louis René Villermé (1782-1863) ${ }^{9}$, a quien sucederán reconocidos científicos y reformistas sociales como Adolphe Focillon (1823-1890), Jules Michel (muerto en 1901), Victor Brants (1836-1917) o Emile Cheysson (1836-1910).

Le Play fue adquiriendo cada vez mayor prestigio; el año 1862 dirigió la sección francesa de la Exposición de Londres y en 1864 publicará La réforme sociale en France ${ }^{10}$, que tuvo nada menos que seis ediciones en vida del autor y fue muy alabada por destacadas figuras del momento como Sainte-Beuve, Montalembert o Albert Schäffle ${ }^{11}$. Para la siguiente exposición francesa, el año 1867, Le Play fue nombrado de nuevo comisario general y el éxito fue tan colosal que el emperador le designó senador. No debemos olvidar la importancia que tenían estas exposiciones universales para el prestigio de una nación. Las de París simbolizaban la prosperidad de la burguesía francesa del Segundo Imperio que rivalizaba con la Inglaterra victoriana.

Le Play participó activamente en el gobierno provisional de 1848 y en el Segundo Imperio con el fin de conseguir poner en práctica sus planes de reforma social. En estos años, su notoriedad en el campo de las ciencias técnicas y en el de la incipiente sociología era muy reconocida. En realidad, es en este cruce de múltiples caminos entre las revoluciones políticas y las industriales, entre la Ilustración y la crítica tradicionalista, donde se sitúa el nacimiento de la sociología.

\section{Los últimos años}

En la última etapa de su vida, a partir de 1870, Le Play se retira de la vida política y se dedica de lleno a su obra de reforma social, centrada en su sociedad de estudios sociales, y reafirmándose en la creencia de que la única vía de reforma

7. Solicitud que publica Les Études Sociales, núm. 129, p. 75, primer semestre 1999. Presentada por E. Secretan y A. Savoye.

8. Cuatro de las cartas de Le Play a Ollivier han sido publicadas en Les Études Sociales, núm. 120 , p. 1-4, 1992. Sobre la relación entre Le Play y Ollivier, puede verse A. SAVOYE, «Le Play, conseiller d'Emile Ollivier», Les Études Sociales, núm. 120, p. 5-11, 1992.

9. El estudio de Villermé sobre las clases obreras fue pionero y tuvo mucha repercusión. Su título es: Tableau de l'état physique et morale des ouvriers employés dans les manufactures de coton, de laine, et de soie. Fue publicado en dos volúmenes, en París, por Renouard, en el año 1840.

10. Su título completo es: La réforme sociale en France, déduite de l'observation comparée des peuples européens.

11. Fragmentos de estos autores se encuentran en la introducción de La réforme sociale en France de las sucesivas ediciones. 
social será la difusión de sus estudios. Por ello se dedica a completar o a condensar sus teorías en distintas obras: L'Organisation de la famille (1871), La paix sociale après le désastre (1871), La correspondance sociale (1872), La constitution de l'Angleterre (1875), La réforme en Europe et le salut en France (1876), La question sociale au XIX siècle (1879), La méthode de la science sociale (1879) y por último, La constitution essentielle de l'humanité (1881). Además, aparece la segunda edición, en seis volúmenes, de Les ouvriers européens (1877-79) y seis de La réforme sociale en France. Hasta su muerte, Le Play supervisó las monografías de Les ouvriers des deux mondes y de los boletines anuales de la Société d'Économie Sociale.

Todas sus obras se agrupaban en la denominada Bibliothèque Sociale, la cual constituía el edificio doctrinal leplayano y era utilizada por el propio Le Play para formar a sus nuevos seguidores y colaboradores. Esta formación se realizaba, o bien a través de unos cursos, que dirigió durante muchos años un destacado discípulo de Le Play, Adolphe Focillon, o por medio de la Société d'Économie Sociale y de las Unions de la Paix Sociale, fundadas éstas en 1872, desde una perspectiva más práctica, divulgativa y con la clara intención de captar el máximo número de miembros.

Desencantado ante la idea de que el Estado y las instituciones políticas fueran capaces de asumir la reforma social, Le Play cree firmemente que esta tarea le corresponde, más que nunca, a una clase superior que esté por encima de las rivalidades políticas. Con el objetivo de constituir esa clase superior que debería hacerse cargo de impulsar la reforma social, Le Play funda las Unions de la Paix Sociale, cuya misión será, fundamentalmente, formar y enseñar a esas nuevas elites, así como propagar los estudios sociales y despertar afición a ellos y, de este modo, trabajar para la prosperidad de las sociedades. Así es como Le Play introdujo la práctica de la investigación de equipo. Las Unions de la Paix Sociale consiguen que Le Play se vea rodeado de personas que se adhieren a su método y su doctrina. Entre estos discípulos destacan Adolphe Focillon, Emile Cheysson, Jules Michel, Claudio Jannet (1844-1894) y Alexis Delaire (1836-1915), quienes constituirán la primera union, la cual será modelo de todas las que se establezcan, tanto en Francia como en otros países. Pronto hubo unions por toda Francia y en muchos otros países, destacando Bélgica y Suiza. En el año 1874 eran 209 miembros; en 1875, 416 miembros; en 1876, 918 miembros; en 1884, 2731 miembros y en el año 1885, las unions contabilizaban un total de 3253 miembros $^{12}$.

Por último, la revista La Réforme Sociale, cuyo primer número vio la luz en el mes de enero del año 1881, un año antes de la muerte de Le Play, se encargará de difundir y popularizar las actividades de todo el grupo.

Frédéric Le Play recibirá en el salón de su domicilio hasta la fecha de su fallecimiento, en 1882. Todos los lunes, de ocho a diez y media de la noche, acuden a la parisina plaza de Saint-Sulpice, número seis, además de los discípulos de Le Play, las personalidades más relevantes de la ciencia, la política, la

12. Según A. SaVOYe, 1989, p. 45. 
industria y la economía social y política, ya fueran franceses o venidos de cualquier país europeo ${ }^{13}$, ocurriendo, a veces, que se escuchaban cinco o seis idiomas a la vez. En estas reuniones se preparaban las sesiones mensuales de la Société d'Économie Sociale, pero, además, se leía en voz alta, se jugaba a las cartas y se mantenían conversaciones serias o alegres, dependiendo de los visitantes $^{14}$. Igualmente, llegaban a este salón jóvenes que querían consultar con Le Play, a la manera de un médico social, aspectos concretos de la ciencia de la sociedad $^{15}$. A la muerte de Le Play, un cronista del diario Le moniteur universel describía del siguiente modo el conocido salón:

Está vacío, desde ahora, este gran salón de la plaza Saint-Sulpice que ha visto pasar tantos hombres ilustres y tantas cosas, donde la gloria oficial y aquella que no lo es, los talentos y las condiciones más diversas se codeaban en un original revoltijo. Un miembro de la Cámara alta de Inglaterra contestaba a un alumno de la Escuela de Minas, un obispo francés charlaba con un turco de la embajada otomana. Se hablaba cinco o seis lenguas a la vez ${ }^{16}$.

De este modo, igual que cualquier otra velada transcurrió la del 4 de abril de 1882. Sin embargo, al día siguiente, alrededor del mediodía, Le Play perdió súbitamente el conocimiento y murió sin dolor poco después.

\section{Bibliografía}

ARnault, Françoise (1993). Frédéric Le Play. De la métallurgie à la science sociale. Nancy: Presses Universitaires de Nancy.

Brooke, Michael Z. (1970). Le Play: Engineer and Social Scientist. Londres: Longman.

DuparC, A. (1882). «F. Le Play et les jugements de la presse», La Réforme sociale: 351 360 y $430-438$.

Chapelle-Duliere, Jacqueline (1989). «Les salons de Frédéric Le Play», Sociétés, núm. 23, mayo.

FOURCY, Lefébure (1883). «P. G. F. Le Play: notice biographique», La Réforme sociale, 1: 29-44.

Jardin, André (1984). Alexis de Tocqueville. París: Hachette.

KalaOra, Bernard; Antoine SAVOYE (1989). Les inventeurs oubliés: Le Play et ses continuateurs aux origines des sciences sociales. Seyssel: Champ Vallon.

Le PlaY, Albert (1956). «Souvenirs sur F. Le Play», en Recueil d'Études Sociales publié à la mémoire de Frédéric Le Play. París: Picard.

LE PLAY, Frédéric (1855). Les ouvriers européens. Études sur les travaux, la vie domestique et la condition morale des populations ouvrières de l'Europe, précédées d'un exposé de la méthode d'observation. París: Impr. Impériale.

- (1864). La réforme sociale en France, déduite de l'observation comparée des peuples européens. París: Plon. 2 vols.

13. Albert Le Play, 1956, p. 9.

14. LefÉbure De FourCY, 1883, p. 44.

15. Pierre Méline, 1912, p. 3.

16. Recogido por A. DUPARC, 1882, p. 359. 
- (1877-1879). Les ouvriers européens. Études sur les travaux, la vie domestique et la condition morale des populations ouvrières de l'Europe, précédées d'un exposé de la méthode d'observation. $2^{\text {a }}$ ed. Tours: A. Mame et fils. 6 vols.

Meline, Pierre (1912). F. Le Play: L’Oeuvre de science. París: Bloud et Gay.

SavoYe, Antoine (1989). "Le Play et la méthode sociale», en F. Le PlaY, La méthode sociale. París: Méridiens Klincksieck.

— (1992). «Le Play, conseiller d'Emile Ollivier», Les études sociales, núm. 120: 5-11. 